OMAE2014-23843

\title{
EFFECTS OF TOP-END VESSEL HEAVE ON SUBMARINE RISER VIV OF DEEP WATER PLATFORM
}

\author{
Weimin Chen* \\ Key Laboratory for Mechanics in Fluid Solid \\ Coupling System, Institute of Mechanics , \\ Chinese Academy of Sciences \\ Beijing , 100190, China
}

\author{
Shuangxi Guo \\ Key Laboratory for Mechanics in Fluid Solid \\ Coupling System, Institute of Mechanics, \\ Chinese Academy of Sciences \\ Beijing , 100190, China
}

\author{
Min Li \\ School of Aeronautics Sciences and Engineering, \\ Beijing University of Aeronautics and Astronautics \\ Beijing 100191, China
}

\begin{abstract}
The dynamic coupling between moving top-end vessel and submarine riser becomes more remarkable for a floating platform in deeper water due to the larger top-end motion amplitude, compared with the fixed platform in shallow water. In this study the impacts of top-end heave on the riser undergoing vortex-induced vibration (VIV) are explored in terms of the parametric excitation and the consequent dynamic behaviors. By using finite element simulations based on a coupled hydrodynamic force approach, the dynamic responses of the integrated system including both a floating top-end and the riser experiencing VIV are examined.

Our numerical results show that the riser displacement becomes several times larger than the displacement for the case without top-end motion, and the impact of heave on riser VIV response gets larger as the modal order number dropping. Riser VIV amplitude becomes, almost linearly, more profound when the tension ratio, as one of critical parameters that influence the riser dynamic response, gets larger. Moreover, an interesting phenomenon called mode transition is observed, particularly at lower frequency, during modal dynamics response.
\end{abstract}

\section{INTRODUCTION}

For platform in deepwater, marine risers are usually employed to convey gas and oil or optical and electrical information between top-end vessel and sea bed. Consequently, the vortex-induced vibration (VIV) of slender risers with large aspect ratio becomes more complicated as water depth increasing. For example, the shedding modes or frequencies of wake-vortex may vary along the riser length rather than keeping constant. Additionally, the dynamic characteristics of slender riser usually presents low-frequency and high-density natural modes due to its large structural flexibility. Therefore, the VIV of a slender riser often exhibits new phenomena ${ }^{[1,2]}$ such as multi-mode VIV, travelling wave and wide-band random vibrations, which have presented new challenges to researchers.

On the other hand, compared with fixed platform in shallow water, floating platform in deep water has larger motion amplitude, and the coupling between top-end vessel and submarine riser appears to be more pronounced. Moreover, new issues such as additional lock-in region, parametric excitation and response amplification owing to the coupling are introduced $^{[3-5]}$. It is noted that the tension fluctuation due to top-end heave may cause a riser VIV involving higher-order modes along with larger-amplitude dynamic response, e.g. 10\% 
and 20 100\% higher riser displacement and shear stress respectively than the case without vessel motion ${ }^{[5]}$. Moreover, Silveira $^{[6]}$ (2007) found mode jump may occur during the dynamic response. He investigated the effect of vertical motion of the floating unit on the VIV of risers by using a finite element model coupled to a wake-oscillator model. Park and Jung $^{[7]}$ (2002) reported that the parametric excitation alters the response pattern of a long slender marine structure.

Among the researches on the dynamic coupling between top-end vessel and marine riser, most of them address on topend dynamic response. Generally, the methods of those researches can be classified into two kinds: quasi-static $\operatorname{method}^{[8-11]}$ and coupled method ${ }^{[12-18]}$. In quasi-static method, riser is modeled by a spring with lumped mass, and only hydrostatic restoring force of riser is considered. In coupled method, submarine riser and its hydrodynamic force are mostly simplified $^{[15,17,18]}$, e.g. the Morison formula is employed to model hydrodynamic force. Moreover, the main concern of previous researches is dynamic responses of riser rather than VIV of riser.

In addition, it is worthwhile to mention that the dynamic coupling mechanisms, between top vessel and submarine riser, owing to different vessel motions are essentially different. Taking horizontal motion, i.e. sway or surge of vessel, as an example, the transverse vibration of top-end propagates along riser. This transverse vibration may directly interact with riser's VIV. Even, the vibrating boundary condition introduced by topend motion might cause nonlinearly coupling such as response amplification or new lock-in. However, if vessel heave is considered, it introduces not only a moving boundary, but more essentially a fluctuating tension of riser. This time-varying tension, in fact, presents a periodically varying structural property, thus, the consequence may be parametric excitation of riser $^{[3,19,20,21]}$. When it comes to parametric excitation, most researches addressed on stability region of time-varying system, e.g. theoretical solutions of stability region based on different theories ${ }^{[3,19,22,23]}$ or the dynamic response of an Euler beam with simplified hydrodynamic force mode ${ }^{[24,25]}$. Park and Jung $^{[7]}$ (2002) implemented a numerical analysis of lateral responses of a long slender marine structure under combined parametric and forcing excitations. Their results demonstrated that a combined excitation needs to be considered for the accurate dynamic analysis of long slender marine structures subjected to a surface vessel motion.

In this paper, the dynamic interaction between top-end vessel heave and riser VIV is considered. First, we develop a hydrodynamic approach to model the vortex-induced lift force which depends on simultaneous structure motion. Then the dynamic response of the integrate system including top-end vessel heave and riser VIV are examined by means of finite element simulations. The effects of top-end heave amplitude and frequency on riser's response displacement as well as vibration propagation are examined so as to have a deeper insight into the interaction between top-end vessel heave and riser VIV.

\section{COUPLING SYSTEM MODEL OF TOP-END HEAVE AND RISER VIV}

\subsection{Parametric Excitation Introduced by Top-End Heave}

Top-end vessel heave essentially introduces a time-varying structural property, a fluctuating tension of riser. This periodically varying structural property consequently causes parametric excitation of the riser. Here, we would first have a theoretical analysis of the dynamic behavior of an Euler Beam pinned at two ends. The governing equation of the Euler beam is

$$
E I \frac{\partial^{4} x(z, t)}{\partial z^{4}}-\left(T_{0}+T \cos \omega_{0} t\right) \frac{\partial^{2} x(z, t)}{\partial z^{2}}+m_{s} \frac{\partial^{2} x(z, t)}{\partial t^{2}}=0
$$

where $x(z, t)$ is the displacement of the beam, and $z$ and $t$ are axial location and time respectively. $E I$ is the bending stiffness. $T_{0}$ is the constant top tension and $T$ and $\omega_{0}$ are the amplitude and frequency of fluctuating tension respectively. $m_{s}$ is the structural mass per unit length. The solution of Eq.(1) is assumed as $x(z, t)=q_{j}(t) \sin \frac{j \pi z}{l}, j=1,2,3 \ldots$. Then we have the Mathieu equation as

$$
\ddot{q}_{j}+\omega_{j}^{2}\left(1-2 \tilde{T} \cos \omega_{0} t\right) q_{j}=0
$$

where

$$
\omega_{j}=\left(\frac{j \pi}{l}\right)^{2}\left(\sqrt{\frac{E I}{m_{s}}+\frac{T_{0} l^{2}}{(j \pi)^{2} m_{s}}}\right), \quad j=1,2,3 \ldots
$$

is the natural frequency of the Euler with constant tension $T_{0}$, and $\tilde{T}=\frac{T}{2\left(T^{*}+T_{0}\right)}, T^{*}=\left(\frac{j \pi}{l}\right)^{2} E I$. If we set $q_{1}(t)$ and $q_{2}(t)$ as two particular solutions, with period $t_{0}$, of Eq.(2), then its eigen-equation can be written as

$$
\lambda^{2}-2 \lambda r+p=0
$$

where $\quad r=\frac{1}{2}\left[q_{1}\left(t_{0}\right)+\dot{q}_{2}\left(t_{0}\right)\right], \quad p=q_{1}\left(t_{0}\right) \dot{q}_{2}\left(t_{0}\right)-q_{2}\left(t_{0}\right) \ddot{q}_{1}\left(t_{0}\right)$ and $\lambda$ is the eigen-root.

As we know, to determine the stability boundary of the Mathieu equation, Eq. (2a), means to get the solution, with a $t_{0}$ or $2 t_{0}$ period, of Eq. (3). When $|r|=1$, and $\lambda_{1}=\lambda_{2}= \pm 1$, the periodic solutions of Eq.(3) are respectively as follows:

$$
\begin{aligned}
& q(t)=d_{0}+\sum_{k=2,4,6 \ldots}^{\infty}\left(c_{k} \cos \frac{k \omega_{0} t}{2}+d_{k} \sin \frac{k \omega_{0} t}{2}\right) \\
& q(t)=\sum_{k=1,3,5 \ldots}^{\infty}\left(c_{k} \cos \frac{k \omega_{0} t}{2}+d_{k} \sin \frac{k \omega_{0} t}{2}\right)
\end{aligned}
$$

Substituting Eq. (4a) and (4b) into Eq. (3) yields:

$$
\left(1-\frac{\omega_{0}^{2}}{4 \omega_{j}^{2}}\right)\left(1-\frac{9 \omega_{0}^{2}}{4 \omega_{j}^{2}}\right)\left(1-\frac{25 \omega_{0}^{2}}{4 \omega_{j}^{2}}\right) \cdots=0
$$




$$
\left(1-\frac{\omega_{0}^{2}}{\omega_{j}{ }^{2}}\right)\left(1-\frac{16 \omega_{0}^{2}}{4 \omega_{j}{ }^{2}}\right)\left(1-\frac{36 \omega_{0}^{2}}{4 \omega_{j}{ }^{2}}\right) \cdots=0
$$

Combining above two equations, we get:

$$
\omega_{0}=\frac{2 \omega_{j}}{k}, k=1,2,3 \ldots
$$

Eq. (6) indicates that a resonance may occur if both excitation frequency $\omega_{0}$ and natural frequency $\omega_{j}$ meet Eq.(6), of which the amplitude will approach infinite if the system damping is zero. Based on above analysis on stability, though, that is done for an Euler beam, further researches ${ }^{[23,26]}$ were generalized to other cases. Tang ${ }^{[23]}$ (2001) classified all resonances into three kinds, i.e. the primary resonance if $\tilde{\omega}=1$ ( $\varpi=\omega_{i} / \omega_{0}$ ), the sub-resonance if $\tilde{\omega}=1 / n(n=2,3 \ldots)$ and the super-resonance if $\tilde{\omega}=n(n=2,3 \ldots)$.

It is more complicated if two excitation frequencies of both top-end heave and vortex-induced lift force are involved. Wang (1998) theoretically studied an Euler beam undergoing top-end heave along with VIV. He pointed out that resonances may occur when three frequencies satisfy $\omega_{v} \pm k \omega_{0}=\omega_{j}, k=0,1,2,3 \ldots$, where $\omega_{v}$ is vortex shedding frequency. As for a practical system, in order to study its dynamic response, finite element simulation is a better alternative, which is more powerful when it comes to a complex system with external loads involving fluid-structure coupling or random distribution. In this study, by developing an approach based on FEM (finite element method) simulation, we will explore the impacts of top-end heave on riser's dynamic response.

\subsection{Numerical Model of Dynamic Response Analysis for the Coupling System}

\subsubsection{Structure Model}

The integrate system including both the top-end vessel and riser is shown in Fig.1.

In Fig. 1a, the origin point of the coordinate system is located at the bottom end of the riser (fixed to the sea bed). The direct of flow $U$ is along the axis $y$. The cross-flow VIV, i.e. riser's vibration along axis $x$, is considered here in this paper, because its vibration amplitude is larger than that of inline VIV. The heave motion of top vessel, $b(t)=B e^{-i \omega_{0} t}$, is along the axis $z$, where $B$ and $\omega_{0}$ are respectively the amplitude and frequency of motion of top-end heave. In the finite element model (shown in Fig.1b), the vessel and the riser respectively consist of 3D cubic solid and 1D Euler beam elements. The rotational motions around the axis $x, y$, and $Z$ of all grids of the top-end are constrained during the FEM calculations so as to avoid a probable singularity introduced by the extremely large mass of the top-end relative to the riser's mass. Additionally, the multi-point constrain (MPC) is used at the joint grid connecting the top-end vessel and the riser, where different elements meet together, so that the constrains can be exerted smoothly upon different freedoms of degrees.

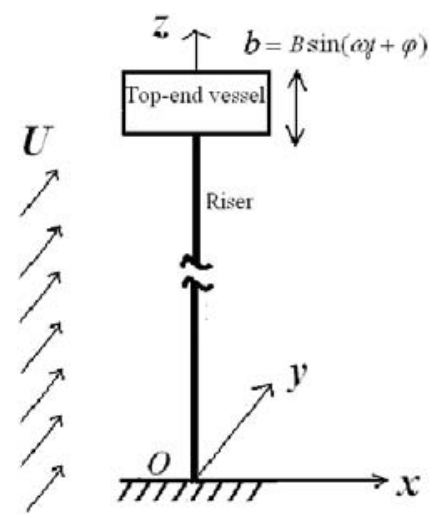

Fig. 1a The platform-riser system and current distribution

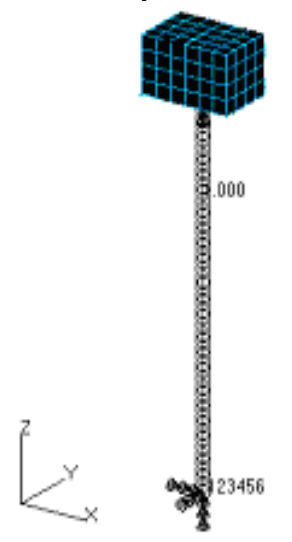

Fig. $1 \mathrm{~b}$ The finite element model of platform-riser system Figure 1 The platform-riser system sketch

\subsubsection{Hydrodynamic Force Model}

The hydrodynamic force $F(z)$, exerted by the ambient

flow around the riser, consists of two parts, i.e. the vortexinduced lift force $F_{L}$ and the fluid drag force $F_{D}$. The fluid drag force $F_{D}$ can be expressed by the Morison equation as

$$
F_{D}=\frac{1}{2} C_{D} \rho_{f} D \dot{x}|\dot{x}|+\frac{1}{4} C_{a} \rho_{f} \pi D^{2} \ddot{x}
$$

where $\rho_{f}$ is the fluid density. $D$ is the riser's outer diameter. $C_{D}$ and $C_{a}$ are the coefficients of drag forces and added mass respectively, of which the values are $C_{a}=1.0$ and $C_{D}=1.1$ for a flexible riser with large aspect ratio.

VIV has always been a challenging issue concerning the interaction between fluid and structural dynamics. It exhibits some interesting, and even unexplained until now, traits like self-excitation, self-limitation of response amplitude, a variety of vortex-shedding modes, multi-mode or wide-band random vibration. Initially, vortex-induced lift force per unit length of riser is somewhat similar with the Morison equation and written as 


$$
F_{L}=\frac{1}{2} \rho_{f} U^{2} C_{L} D
$$

where the lift coefficient $C_{L}$ is a constant value ranging form 0.8 to 1.2 . With recently increasing amount of experimental observations along with CFD simulations, deeper understandings of VIV have been presented. New approaches of hydrodynamic force during lock-in were proposed, which are more accurate and reasonable because of considerations of coupling between structural and fluid dynamics ${ }^{[2,27,28]}$. Sarpkaya ${ }^{[1]}$ (2004) experimentally measured the Fourier average of hydrodynamic force over many cycles of vibration. He decomposed the lift force into two parts, the drag part and the inertia part, which are respectively related to velocity and acceleration of a moving cylinder. He pointed out that for practical Reynolds numbers, the nonlinear expression in manners of structural motion is able to capture the hydrodynamic feature of VIV better than the linear expression. Gopalfrishnan $^{[28]}$ (1993) and Govardhan ${ }^{[29]}$ (2004) implemented a large amount of VIV experiments and presented the lift coefficient in ways of structural motion. Vandiver ${ }^{[2]}$ (2002) suggested that a piecewise parabola function of structural amplitude could be used for the industrial model of lift force to calculate the riser displacement by using the wake oscillator model. Based on above studies, we suggest that lift coefficient $C_{L}$ depend on structure motion rather than being merely a constant value.

A third-order polynomial of the structure velocity is used to model the lift force so that the nonlinear interaction between structural and fluid dynamics is taken into account, i.e.

$$
\begin{aligned}
F_{L}(x) & =\frac{1}{2} \rho U^{2} D\left(C_{L 0} \sin (\omega t)+C_{1} \dot{x}(z, t)+C_{2} \dot{x}^{2}(z, t)+C_{3} \dot{x}^{3}(z, t)\right) \\
& =p_{f} C_{L}(\dot{x}(z, t))
\end{aligned}
$$

where $\quad p_{f}=\frac{1}{2} \rho U^{2} D$ and the lift coefficient is $C_{L}(\dot{y}(z, t))=C_{L 0} \sin (\omega t)+C_{1} \dot{x}(z, t)+C_{2} \dot{x}^{2}(z, t)+C_{3} \dot{x}^{3}(z, t)$. The values of the coefficients $C_{L 0}, C_{1}, C_{2}$ and $C_{3}$ can be derived by fitting experimental data. Among all VIV experiments, the results obtained in situations, of cylinder freely vibrating or flexible cable (with large aspect ratio) rather than forced vibration or rigid body, are strongly recommended, e.g. experiments of Gopalkrishnan(1993), Trim(2005) and Chaplin(2005) ${ }^{[29-31]}$. Chen (2012) gave an approach to calculate the coefficients' values by fitting experimental data.

Observing Eq. (7), we may say it can capture, to some extent, the features of VIV. 1) The feature of self-excitation. In Eq. (7), the excitation is represented by the first term $p_{f} C_{L 0} \sin (\omega t)$, a sinusoidal excitation force, together with the second term, $p_{f} C_{1} \dot{x}(z, t)$, which increases as response increasing ( $C_{1}$ is required to be positive). 2) The feature of self-limitation. One of unique traits of VIV is that structural response never rises infinitely, but begins to drop when response amplitude reaches to a certain number, such as $\bar{x}_{\max }=1.5$ or 2.0. This feature, called self-limitation, is represented by the nonlinear terms with higher orders in Eq.(7), $\rho_{f} C_{2} \dot{x}^{2}(z, t)$ or $\rho_{f} C_{3} \dot{x}^{3}(z, t)$ (at least one of the coefficients $C_{2}$ and $C_{3}$ is negative). 3) Axially varying distribution of lift force along riser. For case of a rigid cylinder, the vortexinduced lift force uniformly distributes along riser. But for case of a flexible slender riser, the coherence may decrease due to the non-uniform distributions of lift force as well structural motion. In the present model, the lift force is non-uniform because of the axially-varying structural motion. Therefore, the span coherence of flexible riser is automatically captured.

\section{EFFECTS OF TOP-END VESSEL HEAVE ON RISER VIV}

By cooperating the presented hydrodynamic model with the structure model, we carried out the dynamic response calculations of the integrate system (shown in Fig.1) by using the FEM code (Chen et al., 2012). In order to explore the impacts of top-end vessel heave on riser VIV, we will study the riser response displacements and its wave propagations along riser length at different heave frequencies and tension ratios.

The structural parameters of the riser are as follows: the outer and inner diameters are respectively $D=0.500 \mathrm{~m}$ and $d=0.445 \mathrm{~m}$. The riser length is $500 \mathrm{~m}$, and the material density is $\rho_{s}=7.8 \times 10^{3} \quad\left(\mathrm{~kg} / \mathrm{m}^{3}\right)$. The bending stiffness is $E I=3.8 \times 10^{9}\left(\mathrm{Nm}^{2}\right)$ and the structural damping ratio is 0.03 . The top tension is $T=6.8 \times 10^{7}(\mathrm{~N})$. The values of the lift coefficients are $C_{L 0}=0.22, C_{L 1}=0.35, C_{L 2}=-0.67$ and $C_{L 3}=-1.08$ respectively. The velocity and density of flow are $U=1.0 \mathrm{~m} / \mathrm{s}$ and $\rho_{f}=1.0 \mathrm{~kg} / \mathrm{m}^{3}$ respectively. The vortexinduced frequency is assumed consistent with the structural frequency, i.e. $\omega_{v}=\omega_{j}$.

\section{1 Effects of Modal Frequencies}

The riser dynamic responses were simulated as the topend heaving at the riser's natural frequencies of modes ranging from of mode 1 to mode 24, meanwhile the vortex-induced lift force frequency is same as the heave frequency. Selected dynamic response displacements, non-dimensioned by the riser's outer diameter $D$ as $x / D$, are presented in Fig. 2 .

Generally speaking, the response displacements increase as the heave frequencies decreasing. It is also noted that the modal responses of lower-order modes are mostly dominated by standing wave, while obvious travelling wave can been seen in the responses of higher-order modes, see Fig. 2e and Fig. $2 \mathrm{f}$ where there is no exact node. This is mainly because the damping of the modes with higher order number becomes larger, and the modal dynamic response attenuates faster. Thus, the riser vibration may attenuate rapidly into a pretty little, even zero, value before it meet the reflect wave to form a standing wave. Also, with the incease of mode order number, structural 
deformation curvature increases. So, compared with tesion stiffness, effect of bending stifness becomes more significant, in other words, the impact of tension on dynamic response gets smaller.

Observing the displacement responses of the riser undergoing both VIV and top-end heave, see Fig. 2a, we noted that the value of the displacement may get larger than that of VIV alone (the displacement limition of VIV is usually around 1.5 2.0) or,even, that of parametic excitation alone. That is probably because the nonlinear interaction between top-end motion (along with, consequently, the axial motion) and the riser's VIV, which might nonlineally amplify the dynamic response of the riser. Similar phenonmina, a combination of vertical and lateral excitation giving greater amplitude, were successively reported in the references [7,26,32].
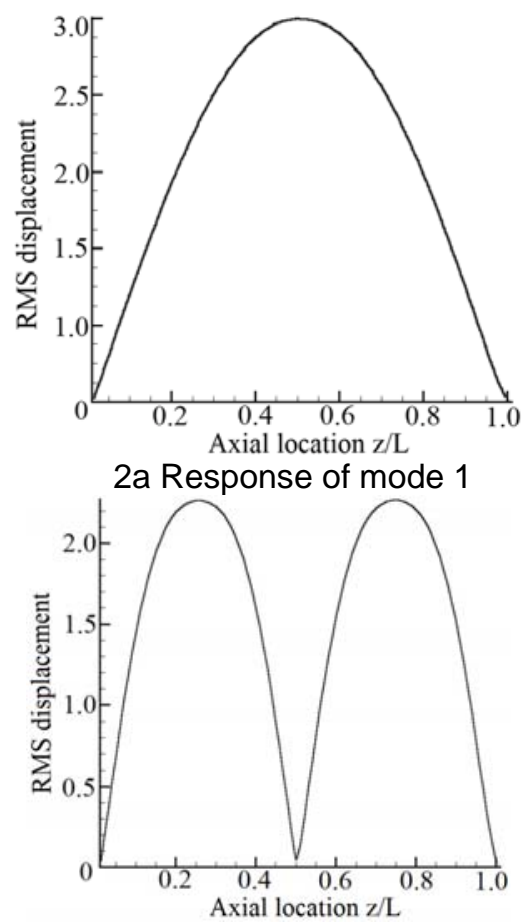

$2 b$ Response of mode 2

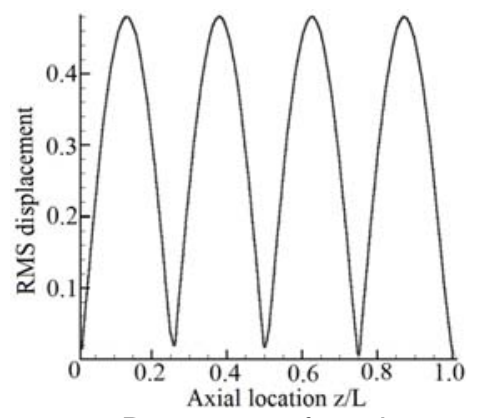

2c Response of mode 4

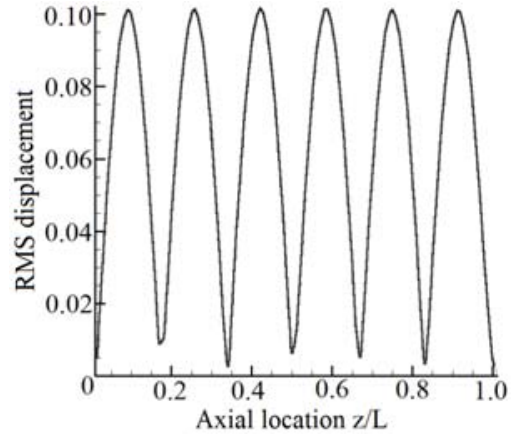

$2 \mathrm{~d}$ Response of mode 6

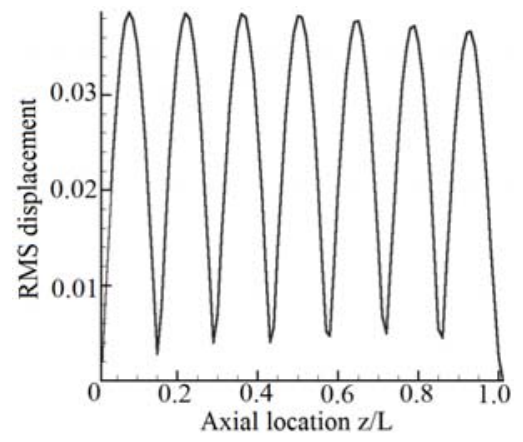

2e Response of mode 7

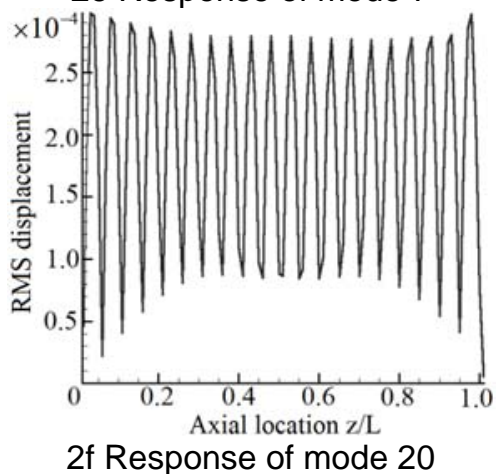

Figure 2 RMS displacement of dynamic response of the riser experiencing both top-end heave and VIV

We also compared the displacement responses of the riser with top-end heave to the case without top-end heave, see Fig. 3. It is shown that the maximum amplitude for the riser experiencing both top-end heave and VIV is larger than the case without top-end motion. Take mode 1 as an example, its maximum amplitude is about 3 times of the case without topend heave. 


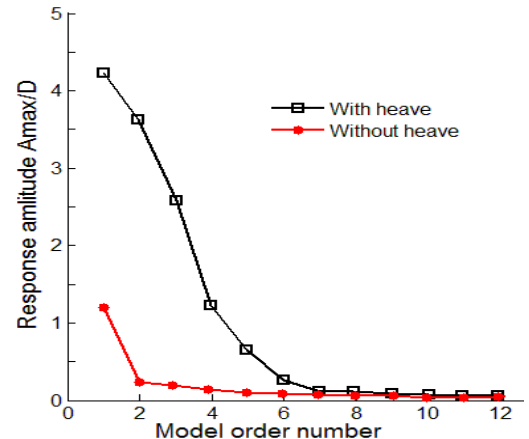

Figure 3 Comparison of modal dynamic responses between cases with top-end heave and without top-end heave

\subsection{At Other Frequencies}

For the cases of top-end heaving at other frequencies, e.g. $\omega_{0}=\omega_{j}, \frac{1}{2} \omega_{j}, \frac{2}{3} \omega_{j}, 2 \omega_{j}, 4 \omega_{j}$, the riser's dynamics responses are presented in Fig. 4.. By comparing Fig.4a and Fig.4b where the heave frequencies are respectively $\omega_{0}=\frac{1}{2} \omega_{1}$ and $\omega_{0}=\frac{2}{3} \omega_{1}$, we note that the dynamic response amplitude changes as the heave frequency changing, though the wave shapes of RMS displacements look alike.

In fig. 4c, parametric excitations occur when the heave frequencies are repectively $\varpi=1,1 / 2$ and 2 , and the dynamice response amplitude is larger than other senarios. Particularly, the largest response amplitude happens at the frequency $\varpi=2$. But for mode 3 , the dynamics responses, see Fig. 5, is somewhat different from mode 1. In Fig. 5c, the largest response amplitude happens at the frequency $\varpi=1$, but not as it is $\varpi=2$, in Fig. 4c.

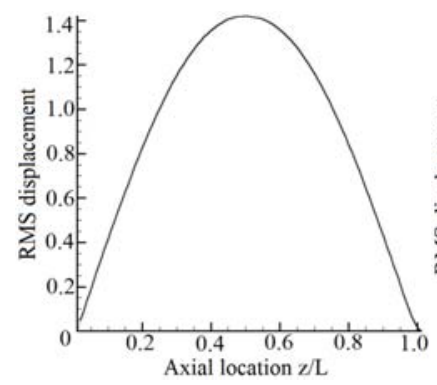

4a RMS displacement at $\frac{1}{2} \omega_{1}$ heave frequency

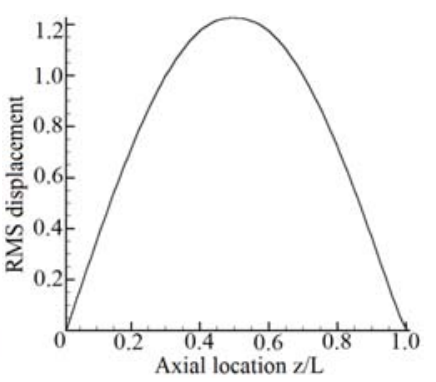

4b RMS displacement at $\frac{2}{3} \omega_{1}$ heave frequency

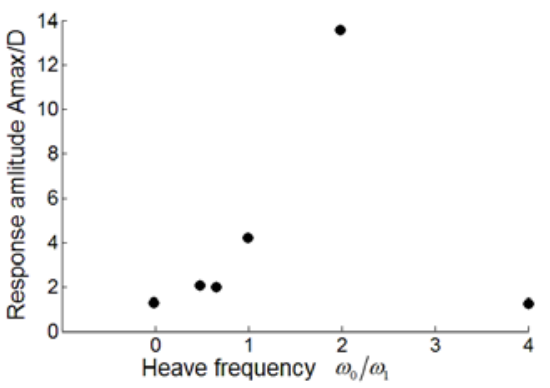

4c Maximum displacement versus heave frequency Figure 4 Dynamic response of mode 1 at different topend heave frequencies

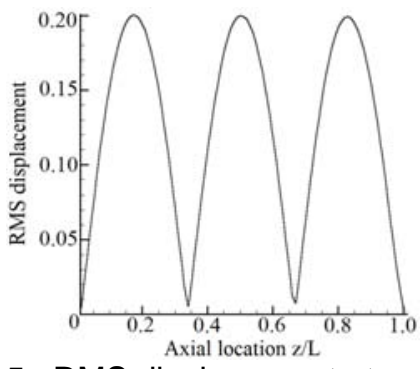

5a RMS displacement at $\frac{1}{2} \omega_{3}$ heave frequency

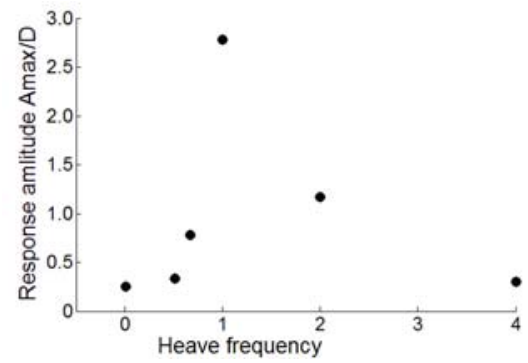

5c Maximum displacement versus heave frequency Figure 5 Dynamic response of mode 3 at different topend heave frequencies

An interesting phenomenon (called mode transition here) is observed during the dynamics responses at some special frequencies. Similar phenomenon (called mode jumps then) is reported by Silveira ${ }^{[6]}$ (2007) who used Hilbert-Huang spectral analysis technique helps distinguishing mode jumps by tracking frequency responses in time. Park and Jung ${ }^{[7]}$ (2002) also pointed out that the parametric excitation may alter the response pattern of a long slender marine structure.

We think the mode transition in our numerical simulations may be introduced by riser's frequency multiplication due to its natural dynamics characteristics. According to Eq.(2b), for the modes with lower modal order number, the frequency value is approximately proportional to the order number $j$ because the value of tension stiffness is much larger than the bending stiffness. In that case, there may be frequency multiplication. 
The natural frequencies of the riser are listed in Table 1 , in which we can see that $\omega_{2} \approx 2 \omega_{1}, \omega_{4} \approx 2 \omega_{2}$. The dynamic responses in terms of RMS displacement and temporal-spatial evolution are present in Fig. 6, Fig. 7 and Fig. 8 respectively for cases of the excitation frequencies are $\omega_{0}=2 \omega_{1}, \omega_{0}=\omega_{2}$ and $\omega_{0}=\omega_{4}$.

\begin{tabular}{cccc}
\multicolumn{2}{l}{ Table 1 Riser's natural frequencies } & unit: $\mathrm{Hz}$ \\
\hline $\begin{array}{c}\text { Mode } \\
\text { number }\end{array}$ & 1 & 2 & 4 \\
frequency/Hz & 0.1715 & 0.3415 & 0.6917 \\
\hline
\end{tabular}

Comparing Fig. 6a and Fig. 7a, we can see that the two RMS displacements look quite alike and hold almost same displacement values. But observing the temporal-spatial evolution of displacement, see Fig. 6b and 7b, we can see an interesting difference. As $\omega_{0}=\omega_{2}$, the response wave transfer from the modal shape of mode 2 to mode 1 , we call it mode transformation here, after a certain periods of time see Fig. 7b. Moreover, the response displacement gets larger after the mode transformation happens. This phenomenon is also observed for case of the excitation frequency $\omega_{0}=\omega_{4}$, that the modal shape transfers from mode 4 to mode 2 while the displacement becoming larger, see Fig. 8 .
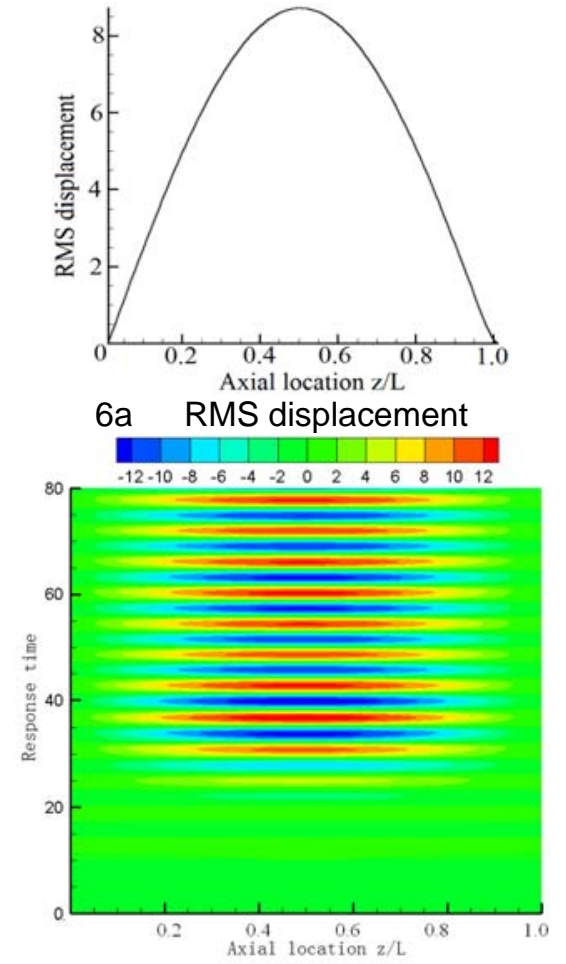

6b Temporal-spatial evolution of response displacement

Figure 6 Dynamic response at top-end heave frequencies, $\omega_{0}=2 \omega_{1}$
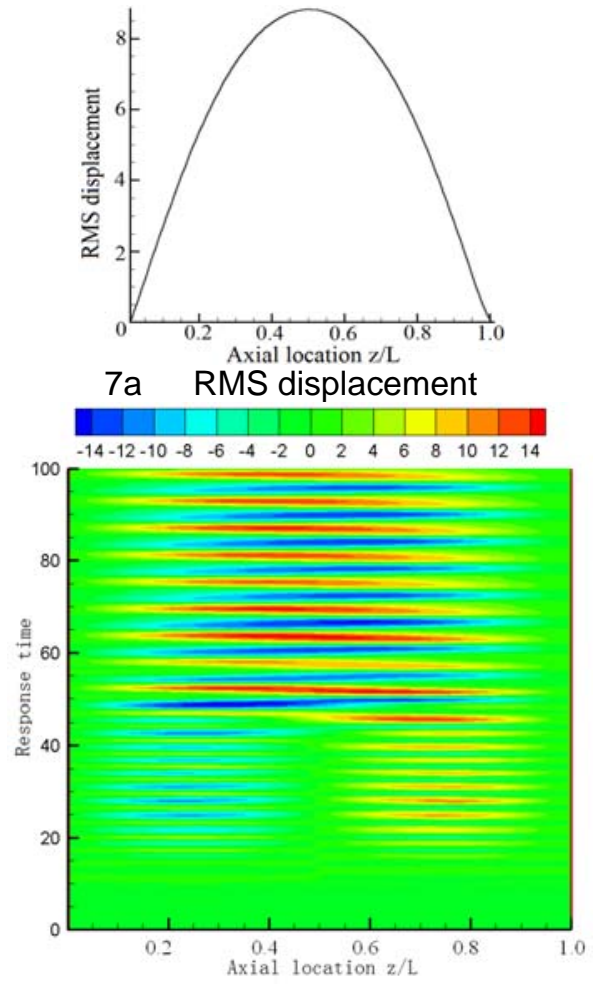

$7 \mathrm{~b}$ Temporal-spatial evolution of response displacement Figure 7 Dynamic response at top-end heave frequencies, $\omega_{0}=\omega_{2}$
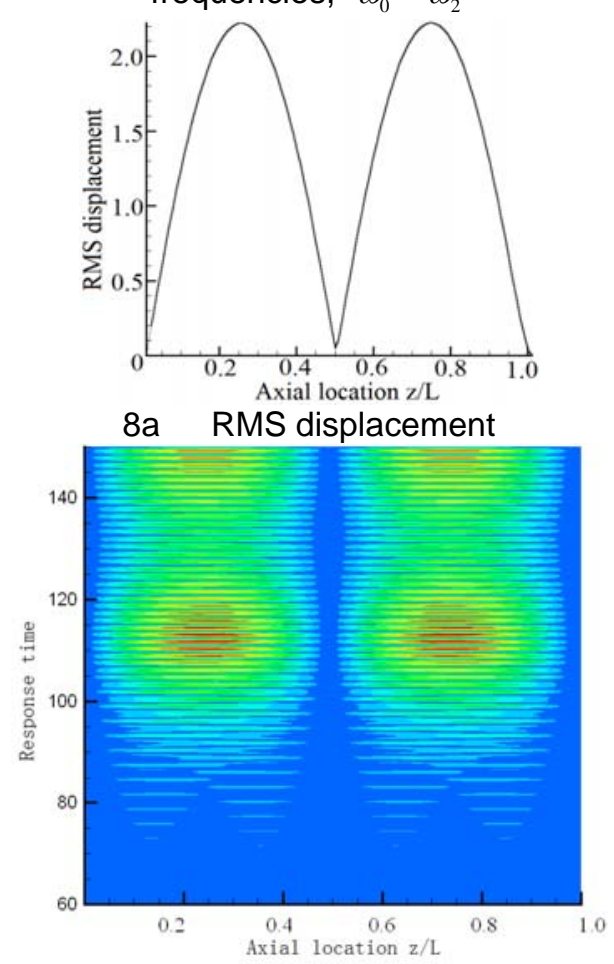

8b Temporal-spatial evolution of response displacement Figure 8 Dynamic response at top-end heave frequencies, $\omega_{0}=\omega_{4}$ 


\subsection{Effects of Tension Ratio}

Effect of tension fluctuation due to top-end heave on riser's dynamic response is examined, and selected results, of mode 1 and mode 3, are presented in Fig. 9. The tension ratio $\bar{T}=T / T_{0}$ is the ratio of tension fluctuation to the constant tension. It is seen, in Fig. 9, that the response displacement gets larger as the tension ratio rising from 0.1 to 1.0. It is also noted that the dynamic response is mostly dominated by standing wave. But as the tension ratio increasing, the dynamics response might be characterized as travelling wave, e.g. the case of $\bar{T}=0.9$ and 1.0 as show in Fig. 9b.

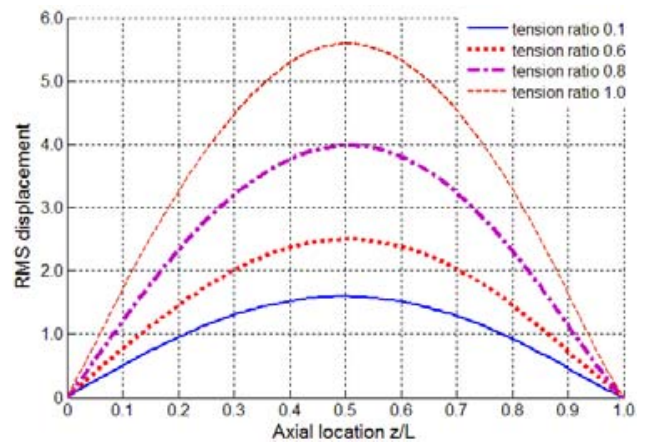

9a RMS displacement response of mode 1

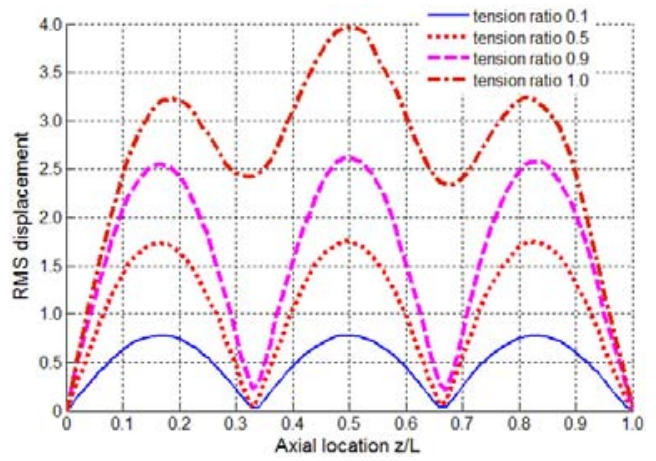

9b RMS displacemen response of mode 3 Figure 9 Dynamics responses at different tension ratios

\section{CONCLUSIONS}

The dynamic responses of the coupling system including both a floating top-end and a riser undergoing VIV are examined by means of finite element simulations. The mechanism of parametric excitation due to top-end heave is firstly theoretically analyzed for an Euler beam. Then, our numerical simulations show following conclusions:

1) The dynamic response amplification gets more pronounced as the number of mode order getting smaller. And, the modal responses of the modes with lower order number are mostly characterized as standing wave, while travelling wave can be seen in the responses of modes with higher order number.

2) Mode transformation is observed during the riser's dynamic responses as the excitation frequency is multi-times value of natural frequency, because of the frequency multiplication of the riser's dynamic characteristics.

3) As the tension ratio increasing, the response displacement gets larger meanwhile the dynamics response might shift from standing wave to travelling wave.

\section{ACKNOWLEDGMENTS}

The authors of this paper would like to thank the financial supports provided by the National Natural Sciences Foundation (Grant No. 11232012 and 11372320).

\section{REFERENCES}

1. Sarpkaya T., 2004. A Critical review of the intrinsic nature of vortex-induced vibration. Journal of Fluids and Structures Mechanics 46, 389-447

2. Vandiver J.K., 2002. A Universal Reduced Damping Parameter for Prediction of Vortex-Induced Vibration. Proceedings of the ASME 21st International Conference on Ocean, Offshore and Arctic Engineering OMAE2002, June 2002, Oslo, Norway

3. Patel MH, and Park HI, 1991. "Dynamics of Tension Leg Platform Tethers at Low Tension. Part I - Mathieu Stability at Large Parameters," Marine Structures, Vol 4: 257-273.

4. Garrett D.L., 2005. Coupled analysis of floating production systems. Ocean Engineering 32, 802-816

5. Wang D.Y., Ling G.C., 1998. Vortex-induced nonlinear vibration of TLP tethers under circumstances of platform oscillation. ACTA Oceannologica SINICA 20(5), 119-128. in Chinese

6. Silveira LMYd., Martins CdA., Leandro DC and Pescee CP. An Investigation on the Effect of Tension Variation on VIV of Risers. Proceedings of the ASME 20th International Conference on Ocean, Offshore and Arctic Engineering OMAE2007-29247, June 2007, San Diego, USA

7. Park HI, and Jung DH, 2002. “A Finite Element Method for Dynamic Analysis of Long Slender Marine Structures under Combined Parametric and Forcing Excitations," Ocean Engineering, Vol 29:1313-1325.

8. Ormberg H., Fylling I.J., Larsen K., Sodahl N., 1997. Coupled analysis of vessel motions and mooring and riser system dynamics. Proceedings of the ASME 16th International Conference on Ocean, Offshore and Arctic Engineering OMAE1997, April 1997, Yokohama, Japan

9. Kim M.H., Arcandra T., Kim Y.B., 2001. Variability of spar motion analysis against various design methodologies/parameters. Proceedings of the ASME 20th International Conference on Ocean, Offshore and Arctic Engineering OMAE2001, June 2001, Rio de Janeiro, Brazil

10. Spanos P.D., Ghosh R., Finn L.D., 2005. Coupled analysis of a spar structure : Monte Carlo and statistical linearization solutions. Journal of Offshore Mechanics and Arctic Engineering 127(1), 11-16. 
11. Wichers J.E.W., Voogt H.J., Roelofs H.W., Driessen P.C.M., 2001. DeepStar-CTR 4401 Benchmark Model Test. Technical Report No. 16417-1-OB, MARIN, Netherlands.

12. Bosman R.L.M., Hooker J., 1999. Elastic modulus characteristics of polyester mooring ropes. Proceedings of the Annual Offshore Technology Conference 1246-1251.

13. Chen X.H., Ding Y., Zhang Y., 2006. Coupled dynamic analysis of a mini TLP: Comparison with measurements. Ocean Engineering 33, 93-117

14. Gu J.Y., Lu H.N., Yang J.M., 2012. Studies on coupling dynamic response and characteristics mooring system of TLP in stochastic waves. The Ocean Engineering 30(4), 42-48. in Chinese

15. Li B.B., Ou J.P., Teng B., 2010. Fully Coupled Effects of Hull, Mooring and Risers Model in Time Domain Based on An Innovative Deep Draft Multi-Spar. China Ocean Engineering 24(2), 219-233.

16. Tahara A., Kim M.H., 2003. Hull/mooring/riser coupled dynamic analysis and sensitivity study of a tanker-based FPSO. Applied Ocean Research 25, 367-382

17. Lee H.H., Wang P.W., 2000. Analytical solution on the surge motion of tension-leg twin platform structural systems. Ocean Engineering 27, 393-415.

18. Tahara A., Kim M.H., 2008. Coupled-dynamic analysis of floating structures with polyester mooring lines. Ocean Engineering 35(17-18), 1676-1685.

19. Chandrasekaran, S, Chandak, NR, and Anupam, G, 2006. "Stability analysis of TLP tethers," Ocean Engineering, Vol 33: 471-482.

20. Chatjigeorgiou, IK, 2004. “On the Parametric Excitation of Vertical Elastic Slender Structures and the Effect of Damping in Marine Applications," Applied Ocean Research, Vol 26: 23-33.

21. Simos A. N. and Pesce, C. P.1997. Mathieu Stability in the Dynamics of TLP's tethers considering variable tension along the Length, Transactions on the Built Enviroment, vol. 29.

22. Han SM, Benaroya H, 2000. "Non-linear Coupled Transverse and Axial Vibration of a Compliant Structure, Part 2: Forced Vibration," Journal of Sound and Vibration, Vol 237(5): 875-900.

23. Tang, J.S., He X.Z., 2001. Response analysis of parametrically excited system. Journal of Yueyang Normal University (Natural Science), Vol. 14 (1):34-40. in Chinese

24. Jian Y.J., E X.Q., Bai W., 2003. Nonlinear Faraday Waves in a Parametrically Excited Circular Cylindrical Container. Applied Mathematics and Mechanics, Vol. 24(10): 60-66.

25. Yang H.Z., Li H.J., 2009. Vibration analysis of deep-sea risers under parametric excitations. Journal of Vibration and Shock. Vol. 28(9): 334-349. in Chinese

26. Xu WH, Zeng XH, Wu YX, 2008. Hill instability analysis of TLP tether subjected to combined platform surge and heave motions. China Ocean Engineering, 22(4): 533-546
27. Chen W.M., Li M., Zheng Z.Q., 2012. Dynamic Characteristics and VIV of Deepwater Riser with Axially Varying Structural Properties. Ocean Engineering 42, 712.

28. Gopalkrishnan R., 1993. Vortex induced forces on oscillating bluff cylinders [Ph.D.Thesis]. MA, USA: MIT, Cambridge.

29. Govardhan R., Williamson C.H.K., 2004. Critical mass in vortex-induced vibration of a cylinder. European Journal of Mechanics B/Fluids 23, 17-27.

30. Trim A.D., Braaten H., Lie H. et. 2005. Experimental investigation of vortex-induced vibration of long marine risers. Journal of Fluids and Structures 21, 335-361.

31. Chaplin, J.R., Bearman, P.W., Huera, F.J., et al. 2005. Laboratory measurements of vortex-induced vibrations of a vertical tension riser in a stepped current. Journal of Fluids and Structures 21, 3-24.

32. Patel MH, and Park HI, 1995. "Combined Axial and Lateral Responses of Tensioned Buoyant Platform Tethers,” Engineering Structures, Vol 17(10): 687-695. 